\title{
On a New Weighted Hilbert Inequality
}

\author{
He Leping, ${ }^{1}$ Gao Xuemei, ${ }^{2,3}$ and Gao Mingzhe ${ }^{2}$ \\ ${ }^{1}$ Department of Mathematics and Applied Mathematics, College of Mathematics and Computer Science, \\ Jishou University, Hunan Jishou 416000, China \\ ${ }^{2}$ Department of Mathematics and Computer Science, Normal College of Jishou University, \\ Hunan Jishou 416000, China \\ ${ }^{3}$ Department of Mathematics, College of Mathematics and Computer Science, Hunan Normal University, \\ Hunan Changsha 410081, China
}

Correspondence should be addressed to Gao Mingzhe, mingzhegao@163.com

Received 13 January 2008; Accepted 18 May 2008

Recommended by László Losonczi

It is shown that a weighted Hilbert inequality for double series can be established by introducing a proper weight function. Thus, a quite sharp result of the classical Hilbert inequality for double series is obtained. And a similar result for the Hilbert integral inequality is also proved. Some applications are considered.

Copyright $(2008$ He Leping et al. This is an open access article distributed under the Creative Commons Attribution License, which permits unrestricted use, distribution, and reproduction in any medium, provided the original work is properly cited.

\section{Introduction}

Let $\left\{a_{n}\right\}$ and $\left\{b_{n}\right\}$ be two sequences of real numbers. It is all known that the inequality

$$
\left(\sum_{n=1}^{\infty} \sum_{m=1}^{\infty} \frac{a_{m} b_{n}}{m+n}\right)^{2} \leq \pi^{2} \sum_{n=1}^{\infty} a_{n}^{2} \sum_{n=1}^{\infty} b_{n}^{2}
$$

is called Hilbert theorem for double series [1], where $\sum_{n=1}^{\infty} a_{n}^{2}<+\infty, \sum_{n=1}^{\infty} b_{n}^{2}<+\infty$, and the constant factor $\pi^{2}$ in (1.1) is the best possible value. And the equality in (1.1) holds if and only if $\left\{a_{n}\right\}$ or $\left\{b_{n}\right\}$ is a zero-sequence. The corresponding integral form of (1.1) is that

$$
\left\{\iint_{0}^{\infty} \frac{f(x) g(y)}{x+y} d x d y\right\}^{2} \leq \pi^{2} \int_{0}^{\infty} f^{2}(x) d x \int_{0}^{\infty} g^{2}(x) d x,
$$

where $\int_{0}^{\infty} f^{2}(x) d x<+\infty, \int_{0}^{\infty} g^{2}(x) d x<+\infty$, and the constant factor $\pi^{2}$ in (1.2) is the best possible value. Recently, various improvements and extensions of (1.1) and (1.2) appear in 
a great deal of papers (see [2-6], etc.). The aim of the present paper is to build some new inequalities by using the weight function method and the technique of analysis, and then to study some applications of them.

First we give some lemmas.

Lemma 1.1. Let $n$ be a positive integer. Then

$$
\int_{0}^{\infty} \frac{d x}{\left(n+x^{2}\right)(1+x)}=\frac{1}{n+1}\left(\frac{\pi}{2 \sqrt{n}}+\frac{1}{2} \ln n\right) .
$$

Proof. Let $a, e, f$ be real numbers. Then

$$
\int \frac{d x}{\left(a^{2}+x^{2}\right)(e+f x)}=\frac{1}{e^{2}+a^{2} f^{2}}\left\{f \ln |e+f x|-\frac{1}{2} \ln \left(a^{2}+x^{2}\right)+\frac{e}{a} \arctan \frac{x}{a}\right\}+C,
$$

where $C$ is an arbitrary constant. This result is given in the paper (see [7]). Based on this indefinite integral it is easy to deduce that the equality (1.3) holds.

Lemma 1.2. If $f(x)=(1 /(x+n))(n / x)^{1 / 2}(1-(\sqrt{x} /(1+\sqrt{x})-\sqrt{n} /(1+\sqrt{n})))$ and $g(x)=$ $(1 /(x+n))(n / x)^{1 / 2}(1+(\sqrt{x} /(1+\sqrt{x})-\sqrt{n} /(1+\sqrt{n})))$, where $n \in N, x \in(0,+\infty)$, then

(1) $f(x)$ and $g(x)$ are monotonously decreasing in interval $(0,+\infty)$;

(2)

$$
\int_{0}^{\infty} f(x) d x=\pi-\pi \omega(n), \quad \int_{0}^{\infty} g(x) d x=\pi+\pi \omega(n),
$$

where the weight function $w$ is defined by

$$
\omega(n)=\frac{\sqrt{n}}{n+1}\left(\frac{\sqrt{n}-1}{\sqrt{n}+1}-\frac{\ln n}{\pi}\right) .
$$

Proof. (1) At first, notice that $1-\sqrt{x} /(1+\sqrt{x})=1 /(1+\sqrt{x})$, hence we can write $f(x)$ in the form of: $f(x)=f_{1}(x)+f_{2}(x)$, where $f_{1}(x)=(1 /(x+n) \sqrt{x})(n /(1+\sqrt{n}))$ and $f_{2}(x)=\sqrt{n} /(x+$ $n)(1+\sqrt{x}) \sqrt{x}$. It is obvious that the functions $f_{1}(x)$ and $f_{2}(x)$ are monotonously decreasing in $(0,+\infty)$. So, $f(x)$ is also monotonously decreasing in $(0,+\infty)$. In the next place, notice that $1-\sqrt{n} /(1+\sqrt{n})=1 /(1+\sqrt{n})$, therefore we can write $g(x)$ in the form of: $g(x)=g_{1}(x)+$ $g_{2}(x)$, where $g_{1}(x)=\sqrt{n} /(1+\sqrt{n})(x+n) \sqrt{x}$ and $g_{2}(x)=\sqrt{n} /(x+n)(1+\sqrt{x})$. It is clear that the functions $g_{1}(x)$ and $g_{2}(x)$ are monotonously decreasing in $(0,+\infty)$. So, $g(x)$ is also monotonously decreasing in $(0,+\infty)$.

(2) Below, we need only to compute the first integral,

$$
\begin{aligned}
\int_{0}^{\infty} f(x) d x & =\int_{0}^{\infty}\left(\frac{1}{x+n}\left(\frac{n}{x}\right)^{1 / 2}\right)\left(1-\left(\frac{\sqrt{x}}{1+\sqrt{x}}-\frac{\sqrt{n}}{1+\sqrt{n}}\right)\right) d x \\
& =\left(1+\frac{\sqrt{n}}{1+\sqrt{n}}\right) \int_{0}^{\infty}\left(\frac{1}{x+n}\left(\frac{n}{x}\right)^{1 / 2}\right) d x-\int_{0}^{\infty}\left(\frac{1}{x+n}\left(\frac{n}{x}\right)^{1 / 2}\right)\left(\frac{\sqrt{x}}{1+\sqrt{x}}\right) d x \\
& =\pi\left(1+\frac{\sqrt{n}}{1+\sqrt{n}}\right)-2 \sqrt{n}\left(\int_{0}^{\infty} \frac{1}{\left(n+t^{2}\right)} d t-\int_{0}^{\infty} \frac{1}{\left(n+t^{2}\right)(1+t)} d t\right) \\
& =\pi-\left\{\pi-2 \sqrt{n} \int_{0}^{\infty} \frac{1}{\left(n+t^{2}\right)(1+t)} d t-\frac{\sqrt{n} \pi}{1+\sqrt{n}}\right\} .
\end{aligned}
$$


He Leping et al.

By Lemma 1.1, we obtain the first integral of (1.5) at once after some simple computations and simplifications.

Similarly, the second integral of (1.5) can be gotten.

Lemma 1.3. Let $\left\{a_{n}\right\}$ be a sequence of real numbers, and let $c(x)$ be a real function and $1-c(n)+c(m) \geq$ $0(n, m \in N)$. If $\sum_{n=1}^{\infty} a_{n}^{2}<+\infty$, then

$$
\sum_{m=1}^{\infty} \sum_{n=1}^{\infty} \frac{a_{m} a_{n}}{m+n}=\sum_{m=1}^{\infty} \sum_{n=1}^{\infty} \frac{a_{m} a_{n}}{m+n}(1-c(n)+c(m)) .
$$

Proof. It is obvious that

$$
\sum_{m=1}^{\infty} \sum_{n=1}^{\infty} \frac{a_{m} a_{n}}{m+n}(1-c(n)+c(m))=\sum_{m=1}^{\infty} \sum_{n=1}^{\infty} \frac{a_{m} a_{n}}{m+n}-\sum_{m=1}^{\infty} \sum_{n=1}^{\infty} \frac{a_{m} a_{n}}{m+n} c(n)+\sum_{m=1}^{\infty} \sum_{n=1}^{\infty} \frac{a_{m} a_{n}}{m+n} c(m) .
$$

We need only to show that

$$
\sum_{m=1}^{\infty} \sum_{n=1}^{\infty} \frac{a_{m} a_{n}}{m+n} c(n)=\sum_{m=1}^{\infty} \sum_{n=1}^{\infty} \frac{a_{m} a_{n}}{m+n} c(m) .
$$

Let $h(m)=\sum_{k=1}^{\infty}\left(a_{k} /(m+k)\right)$. Then

$$
\begin{aligned}
\sum_{m=1}^{\infty} & \sum_{n=1}^{\infty} \frac{a_{m} a_{n}}{m+n} c(m) \\
= & \sum_{m=1}^{\infty}\left(\sum_{n=1}^{\infty} \frac{a_{n}}{m+n}\right) a_{m} c(m)=\sum_{m=1}^{\infty}\left(\sum_{k=1}^{\infty} \frac{a_{k}}{m+k}\right) a_{m} c(m)=\sum_{m=1}^{\infty} h(m) a_{m} c(m)=\sum_{n=1}^{\infty} h(n) a_{n} c(n) \\
= & \sum_{n=1}^{\infty}\left(\sum_{k=1}^{\infty} \frac{a_{k}}{n+k}\right) a_{n} c(n)=\sum_{n=1}^{\infty}\left(\sum_{m=1}^{\infty} \frac{a_{m}}{n+m}\right) a_{n} c(n)=\sum_{n=1}^{\infty} \sum_{m=1}^{\infty} \frac{a_{m} a_{n}}{n+m} c(n)=\sum_{m=1}^{\infty} \sum_{n=1}^{\infty} \frac{a_{m} a_{n}}{m+n} c(n) .
\end{aligned}
$$

Lemma 1.4. Let $\alpha$ be a real number, and let $f(x)$ and $c(x)$ be two real functions, and $\int_{\alpha}^{\infty} f^{2}(x) d x<+\infty$ and $1-c(x-\alpha)+c(y-\alpha) \geq 0$, where $(x, y) \in(\alpha,+\infty) \times(\alpha,+\infty)$. Then

$$
\iint_{\alpha}^{\infty} \frac{f(x) f(y)}{x+y-2 \alpha} d x d y=\iint_{\alpha}^{\infty} \frac{f(x) f(y)}{x+y-2 \alpha}(1-c(x-\alpha)+c(y-\alpha)) d x d y .
$$

Its proof is similar to that of Lemma 1.3. Hence, it is omitted.

\section{Main results}

Theorem 2.1. Let $\left\{a_{n}\right\}$ and $\left\{b_{n}\right\}$ be two sequences of real numbers. If $\sum_{n=1}^{\infty} a_{n}^{2}<+\infty$ and, then

$$
\left(\sum_{m=1}^{\infty} \sum_{n=1}^{\infty} \frac{a_{m} b_{n}}{m+n}\right)^{4} \leq \pi^{4}\left\{\left(\sum_{n=1}^{\infty} a_{n}^{2}\right)^{2}-\left(\sum_{n=1}^{\infty} \omega(n) a_{n}^{2}\right)^{2}\right\}\left\{\left(\sum_{n=1}^{\infty} b_{n}^{2}\right)^{2}-\left(\sum_{n=1}^{\infty} \omega(n) b_{n}^{2}\right)^{2}\right\}
$$

where the weight function $\omega(n)$ is defined by (1.6). 
Proof. Let $c(x)$ be a real function and it satisfies condition $1-c(n)+c(m) \geq 0(n, m \in N)$. First, we suppose that $b_{n}=a_{n}$. By Lemma 1.3 and then applying Cauchy's inequality we have

$$
\begin{aligned}
\left(\sum_{m=1}^{\infty}\right. & \left.\sum_{n=1}^{\infty} \frac{a_{m} a_{n}}{m+n}\right)^{2} \\
& =\left(\sum_{m=1}^{\infty} \sum_{n=1}^{\infty} \frac{a_{m} a_{n}}{m+n}(1-c(n)+c(m))\right)^{2} \\
& =\left(\sum_{m=1}^{\infty} \sum_{n=1}^{\infty}\left(\frac{a_{m}(1-c(n)+c(m))^{1 / 2}}{(m+n)^{1 / 2}}\left(\frac{m}{n}\right)^{1 / 4}\right)\left(\frac{a_{n}(1-c(n)+c(m))^{1 / 2}}{(m+n)^{1 / 2}}\left(\frac{n}{m}\right)^{1 / 4}\right)\right)^{2} \\
& \leq J_{1} J_{2},
\end{aligned}
$$

where

$$
J_{1}=\sum_{m=1}^{\infty} \sum_{n=1}^{\infty} \frac{a_{m}^{2}}{m+n}\left(\frac{m}{n}\right)^{1 / 2}(1-c(n)+c(m)), \quad J_{2}=\sum_{m=1}^{\infty} \sum_{n=1}^{\infty} \frac{a_{n}^{2}}{m+n}\left(\frac{n}{m}\right)^{1 / 2}(1-c(n)+c(m)) .
$$

It is easy to deduce that

$$
J_{1}=\sum_{m=1}^{\infty} \sum_{n=1}^{\infty} \frac{a_{m}^{2}}{m+n}\left(\frac{m}{n}\right)^{1 / 2}(1-c(n)+c(m))=\sum_{n=1}^{\infty}\left(\sum_{m=1}^{\infty} \frac{1}{m+n}\left(\frac{n}{m}\right)^{1 / 2}(1-c(m)+c(n))\right) a_{n}^{2} .
$$

Let $c(x)=\sqrt{x} /(1+\sqrt{x})$. It is obvious that $1-\sqrt{x} /(1+\sqrt{x})+\sqrt{y} /(1+\sqrt{y}) \geq 0$ for $x \geq 0$ and $y \geq 0$. Consider the function $f(x)=\left((1 /(x+n))(n / x)^{1 / 2}\right)(1-(\sqrt{x} /(1+\sqrt{x})-\sqrt{n} /(1+\sqrt{n})))$. By Lemma 1.2, the function $f(x)$ is monotonously decreasing in $(0,+\infty)$. Hereby, we have

$$
\begin{aligned}
J_{1} & =\sum_{n=1}^{\infty}\left(\sum_{m=1}^{\infty} \frac{1}{m+n}\left(\frac{n}{m}\right)^{1 / 2}\left(1-\frac{\sqrt{m}}{1+\sqrt{m}}+\frac{\sqrt{n}}{1+\sqrt{n}}\right)\right) a_{n}^{2} \\
& \leq \sum_{n=1}^{\infty}\left\{\int_{0}^{\infty}\left(\frac{1}{x+n}\left(\frac{n}{x}\right)^{1 / 2}\right)\left(1-\left(\frac{\sqrt{x}}{1+\sqrt{x}}-\frac{\sqrt{n}}{1+\sqrt{n}}\right)\right) d x\right\} a_{n}^{2} .
\end{aligned}
$$

Using (1.5), we can obtain immediately

$$
J_{1} \leq \pi \sum_{n=1}^{\infty} a_{n}^{2}-\pi \sum_{n=1}^{\infty} \omega(n) a_{n}^{2}
$$

Similarly, we have

$$
J_{2} \leq \pi \sum_{n=1}^{\infty} a_{n}^{2}+\pi \sum_{n=1}^{\infty} \omega(n) a_{n}^{2}
$$


He Leping et al.

It follows from (2.2), (2.6), and (2.7) that

$$
\left(\sum_{m=1}^{\infty} \sum_{n=1}^{\infty} \frac{a_{m} a_{n}}{m+n}\right)^{2} \leq \pi^{2}\left\{\left(\sum_{n=1}^{\infty} a_{n}^{2}\right)^{2}-\left(\sum_{n=1}^{\infty} \omega(n) a_{n}^{2}\right)^{2}\right\}
$$

where the weight function $\omega(n)$ is defined by (1.6).

Next, consider the case for $b_{n} \neq a_{n}$. We can apply Schwarz's inequality to estimate the left-hand side of (2.1) as follows:

$$
\begin{aligned}
\left(\sum_{m=1}^{\infty} \sum_{n=1}^{\infty} \frac{a_{m} b_{n}}{m+n}\right)^{4} & =\left\{\left(\int_{0}^{1}\left(\sum_{m=1}^{\infty} a_{m} t^{m-1 / 2}\right)\left(\sum_{n=1}^{\infty} b_{n} t^{n-1 / 2}\right) d t\right)^{2}\right\}^{2} \\
& \leq\left\{\int_{0}^{1}\left(\sum_{m=1}^{\infty} a_{m} t^{m-1 / 2}\right)^{2} d t \int_{0}^{1}\left(\sum_{n=1}^{\infty} b_{n} t^{n-1 / 2}\right)^{2} d t\right\}^{2} \\
& =\left(\sum_{m=1}^{\infty} \sum_{n=1}^{\infty} \frac{a_{m} a_{n}}{m+n}\right)^{2}\left(\sum_{m=1}^{\infty} \sum_{n=1}^{\infty} \frac{b_{m} b_{n}}{m+n}\right)^{2} .
\end{aligned}
$$

And then by using the inequality (2.8), the inequality (2.1) follows from (2.9) at once. It is obvious that the inequality (2.1) is a refinement of (1.1). Below, we give an extension of (1.2).

Theorem 2.2. Let $\alpha$ be a real number, $x \geq \alpha$ and $y \geq \alpha$, and let $f(x)$ and $g(x)$ be two real functions, and $\int_{\alpha}^{\infty} f^{2}(x) d x<+\infty$ and $\int_{\alpha}^{\infty} g^{2}(x) d x<+\infty$. Then

$$
\begin{aligned}
\left\{\iint_{\alpha}^{\infty} \frac{f(x) g(x)}{x+y-2 \alpha} d x d y\right\}^{4} \leq & \pi^{4}\left\{\left(\int_{\alpha}^{\infty} f^{2}(x) d x\right)^{2}-\left(\int_{\alpha}^{\infty} \tilde{\omega}(x) f^{2}(x) d x\right)^{2}\right\} \\
& \times\left\{\left(\int_{\alpha}^{\infty} g^{2}(x) d x\right)^{2}-\left(\int_{\alpha}^{\infty} \tilde{\omega}(x) g^{2}(x) d x\right)^{2}\right\}
\end{aligned}
$$

where the weight function $\tilde{\omega}$ is defined by

$$
\tilde{\omega}(x)= \begin{cases}0, & x=\alpha \\ \frac{x-\alpha}{1+x-\alpha}-\frac{\sqrt{x-\alpha} \ln (x-\alpha)}{\pi(1+x-\alpha)}-\frac{\sqrt{x-\alpha}}{1+\sqrt{x-\alpha}}, & x>\alpha .\end{cases}
$$

Specially, when $\alpha=0$, it is a refinement of (1.2). 
Proof. Let $c(x)$ be a real function, and $1-c(x-\alpha)+c(y-\alpha) \geq 0(x, y) \in(\alpha,+\infty) \times(\alpha,+\infty)$.

Firstly, we suppose that $f=g$. Using Lemma 1.4 and then applying Cauchy's inequality we have

$$
\begin{aligned}
\left\{\iint_{\alpha}^{\infty} \frac{f(x) f(y)}{x+y-2 \alpha} d x d y\right\}^{2} & =\left\{\iint_{\alpha}^{\infty} \frac{f(x) f(y)}{x+y-2 \alpha}(1-c(x-\alpha)+c(y-\alpha)) d x d y\right\}^{2} \\
= & \left\{\iint_{\alpha}^{\infty} \frac{f(x)(1-c(x-\alpha)+c(y-\alpha))^{1 / 2}}{(x+y-2 \alpha)^{1 / 2}}\left(\frac{x-\alpha}{y-\alpha}\right)^{1 / 4}\right. \\
& \left.\times \frac{f(y)(1-c(x-\alpha)+c(y-\alpha))^{1 / 2}}{(x+y-2 \alpha)^{1 / 2}}\left(\frac{y-\alpha}{x-\alpha}\right)^{1 / 4} d x d y\right\}^{2} \\
\leq & \tilde{J}_{1} \tilde{J}_{2},
\end{aligned}
$$

where

$$
\begin{aligned}
& \tilde{J}_{1}=\iint_{\alpha}^{\infty} \frac{f^{2}(x)(1-c(x-\alpha)+c(y-\alpha))}{x+y-2 \alpha}\left(\frac{x-\alpha}{y-\alpha}\right)^{1 / 2} d x d y, \\
& \tilde{J}_{2}=\iint_{\alpha}^{\infty} \frac{f^{2}(y)(1-c(x-\alpha)+c(y-\alpha))}{x+y-2 \alpha}\left(\frac{y-\alpha}{x-\alpha}\right)^{1 / 2} d x d y .
\end{aligned}
$$

In the first place, we consider $\tilde{J}_{1}$ :

$$
\begin{aligned}
\tilde{J}_{1} & =\int_{\alpha}^{\infty}\left\{\int_{\alpha}^{\infty} \frac{1-c(x-\alpha)+c(y-\alpha)}{(x-\alpha)+(y-\alpha)}\left(\frac{x-\alpha}{y-\alpha}\right)^{1 / 2} d y\right\} f^{2}(x) d x \\
& =\int_{\alpha}^{\infty}\left\{\int_{0}^{\infty} \frac{1}{1+u}\left(\frac{1}{u}\right)^{1 / 2} d u\right\} f^{2}(x) d x+\int_{\alpha}^{\infty}\left\{\int_{\alpha}^{\infty} \frac{c(y-\alpha)-c(x-\alpha)}{(x-\alpha)+(y-\alpha)}\left(\frac{x-\alpha}{y-\alpha}\right)^{1 / 2} d y\right\} f^{2}(x) d x \\
& =\pi\left\{\int_{\alpha}^{\infty} f^{2}(x) d x+\int_{\alpha}^{\infty} \tilde{\omega}(x) f^{2}(x) d x\right\},
\end{aligned}
$$

where

$$
\tilde{\omega}(x)=\frac{1}{\pi} \int_{\alpha}^{\infty}\left\{\frac{1}{(x-\alpha)+(y-\alpha)}\left(\frac{x-\alpha}{y-\alpha}\right)^{1 / 2}(c(y-\alpha)-c(x-\alpha))\right\} d y .
$$

We need only to compute the weight function $\tilde{\omega}$. 
He Leping et al.

Let us select still $c(x)=\sqrt{x} /(1+\sqrt{x})$. Then $c(y-\alpha)-c(x-\alpha)=\sqrt{y-\alpha} /(1+\sqrt{y-\alpha})-$ $\sqrt{x-\alpha} /(1+\sqrt{x-\alpha})$. Using (1.4), we have

$$
\begin{aligned}
\tilde{\omega}(x)= & \frac{1}{\pi} \int_{\alpha}^{\infty}\left\{\frac{1}{(x-\alpha)+(y-\alpha)}\left(\frac{x-\alpha}{y-\alpha}\right)^{1 / 2}\left(\frac{\sqrt{y-\alpha}}{1+\sqrt{y-\alpha}}-\frac{\sqrt{x-\alpha}}{1+\sqrt{x-\alpha}}\right)\right\} d y \\
= & \frac{1}{\pi} \int_{\alpha}^{\infty}\left\{\frac{1}{(x-\alpha)+(y-\alpha)}\left(\frac{x-\alpha}{y-\alpha}\right)^{1 / 2}\left(\frac{\sqrt{y-\alpha}}{1+\sqrt{y-\alpha}}\right)\right\} d y \\
& -\frac{1}{\pi} \int_{\alpha}^{\infty}\left\{\frac{1}{(x-\alpha)+(y-\alpha)}\left(\frac{x-\alpha}{y-\alpha}\right)^{1 / 2}\left(\frac{\sqrt{x-\alpha}}{1+\sqrt{x-\alpha}}\right)\right\} d y \\
= & \frac{2}{\pi}(\sqrt{x-\alpha})\left\{\int_{0}^{\infty} \frac{d u}{(x-\alpha)+u^{2}}-\int_{0}^{\infty} \frac{1}{\left((x-\alpha)+u^{2}\right)(1+u)} d u\right\}-\frac{\sqrt{x-\alpha}}{1+\sqrt{x-\alpha}} \\
= & \frac{x-\alpha}{1+x-\alpha}-\frac{(\sqrt{x-\alpha}) \ln (x-\alpha)}{\pi(1+x-\alpha)}-\frac{\sqrt{x-\alpha}}{1+\sqrt{x-\alpha}} .
\end{aligned}
$$

Notice that $\lim _{x \rightarrow \alpha} \tilde{\omega}(x)=0$. Hence, the function defined by (2.11) is just. So,we attain

$$
\tilde{J}_{1}=\pi\left(\int_{\alpha}^{\infty} f^{2}(x) d x+\int_{\alpha}^{\infty} \tilde{\omega}(x) f^{2}(x) d x\right)
$$

Similarly, we have

$$
\tilde{J}_{2}=\pi\left(\int_{\alpha}^{\infty} f^{2}(x) d x-\int_{\alpha}^{\infty} \tilde{\omega}(x) f^{2}(x) d x\right)
$$

We obtain from (2.12), (2.17), and (2.18) that

$$
\left\{\iint_{\alpha}^{\infty} \frac{f(x) f(y)}{x+y-2 \alpha} d x d y\right\}^{2} \leq \pi^{2}\left\{\left(\int_{\alpha}^{\infty} f^{2}(x) d x\right)^{2}-\left(\int_{\alpha}^{\infty} \tilde{\omega}(x) f^{2}(x) d x\right)^{2}\right\}
$$

where the weight function $\tilde{\omega}$ is defined by (2.11). 
Secondly, consider the case for $f \neq g$. We can apply Schwarz's inequality to estimate the left-hand side of (2.10) as follows:

$$
\begin{aligned}
& \left\{\int_{\alpha}^{\infty} \frac{f(x) g(x)}{x+y-2 \alpha} d x d y\right\}^{4} \\
& \quad=\left\{\left(\int_{0}^{\infty}\left(\iint_{\alpha}^{\infty} f(x) g(y) d x d y\right) e^{-u(x+y-2 \alpha)} d u\right)^{2}\right\}^{2} \\
& \quad=\left\{\left(\int_{0}^{\infty}\left(\int_{\alpha}^{\infty} f(x) e^{-u(x-\alpha)} d x\right)\left(\int_{\alpha}^{\infty} g(y) e^{-u(y-\alpha)} d y\right) d u\right)^{2}\right\}^{2} \\
& \quad \leq\left\{\int_{0}^{\infty}\left(\int_{\alpha}^{\infty} f(x) e^{-u(x-\alpha)} d x\right)^{2} d u \int_{0}^{\infty}\left(\int_{\alpha}^{\infty} g(y) e^{-u(y-\alpha)} d y\right)^{2} d u\right\}^{2} \\
& \quad=\left\{\int_{0}^{\infty}\left(\iint_{\alpha}^{\infty} f(x) f(y) d x d y\right) e^{-u(x+y-2 \alpha)} d u \int_{0}^{\infty}\left(\iint_{\alpha}^{\infty} g(x) g(y) d x d y\right) e^{-u(x+y-2 \alpha)} d u\right\}^{2} \\
& \quad=\left\{\left(\iint_{\alpha}^{\infty} \frac{f(x) f(y)}{x+y-2 \alpha} d x d y\right)\left(\iint_{\alpha}^{\infty} \frac{g(x) g(y)}{x+y-2 \alpha} d x d y\right)\right\}^{2} .
\end{aligned}
$$

It follows from (2.19) and (2.20) that the inequality (2.10) is valid.

\section{Applications}

As applications, we will give some new refinements of Hardy-Littlewood's theorem and Widder's theorem below.

Let $f(x) \in L^{2}(0,1)$ and $f(x) \neq 0$ for all $x$. Define a sequence $\left\{a_{n}\right\}$ by

$$
a_{n}=\int_{0}^{1} x^{n} f(x) d x, \quad n=0,1,2, \ldots
$$

Hardy-Littlewood [1] proved that

$$
\sum_{n=0}^{\infty} a_{n}^{2}<\pi \int_{0}^{1} f^{2}(x) d x
$$

where $\pi$ is the best constant that the inequality (3.2) keeps valid.

Theorem 3.1. With the assumptions as the above-mentioned, define a sequence $\left\{a_{n}\right\}$ by $a_{n}=$ $\int_{0}^{1} x^{n-1 / 2} f(x) d x, n=1,2, \ldots$. Then

$$
\left(\sum_{n=1}^{\infty} a_{n}^{2}\right)^{2}<\pi\left\{\left(\sum_{n=1}^{\infty} a_{n}^{2}\right)^{2}-\left(\sum_{n=1}^{\infty} \omega(n) a_{n}^{2}\right)^{2}\right\}^{1 / 2} \int_{0}^{1} f^{2}(x) d x,
$$

where $\omega(n)$ is defined by (1.6). 
He Leping et al.

Proof. By our assumptions, we may write $a_{n}^{2}$ in the form of: $a_{n}^{2}=\int_{0}^{1} a_{n} x^{n-1 / 2} f(x) d x$.

Apply Schwarz's inequality to estimate the left-hand side of (3.3) as follows:

$$
\begin{aligned}
\left(\sum_{n=1}^{\infty} a_{n}^{2}\right)^{2} & =\left(\sum_{n=1}^{\infty} \int_{0}^{1} a_{n} x^{n-1 / 2} f(x) d x\right)^{2} \\
& =\left\{\int_{0}^{1}\left(\sum_{n=1}^{\infty} a_{n} x^{n-1 / 2}\right) f(x) d x\right\}^{2} \leq \int_{0}^{1}\left(\sum_{n=1}^{\infty} a_{n} x^{n-1 / 2}\right)^{2} d x \int_{0}^{1} f^{2}(x) d x \\
& =\int_{0}^{1} \sum_{m=1}^{\infty} \sum_{n=1}^{\infty} a_{m} a_{n} x^{m+n-1} d x \int_{0}^{1} f^{2}(x) d x=\left(\sum_{m=1}^{\infty} \sum_{n=1}^{\infty} \frac{a_{m} a_{n}}{m+n}\right) \int_{0}^{1} f^{2}(x) d x .
\end{aligned}
$$

It is known from (2.8) and (3.4) that the inequality (3.3) is valid. Theorem is therefore proved.

Let $a_{n} \geq 0(n=0,1,2, \ldots), A(x)=\sum_{n=0}^{\infty} a_{n} x^{n}$, and $A^{*}(x)=\sum_{n=0}^{\infty}\left(a_{n} x^{n} / n !\right)$. Then

$$
\int_{0}^{1} A^{2}(x) d x \leq \pi \int_{0}^{\infty}\left(e^{-x} A^{*}(x)\right)^{2} d x
$$

This is the famous Widder theorem (see [1]).

Theorem 3.2. With the assumptions as the above-mentioned, then

$$
\left(\int_{0}^{1} A^{2}(x) d x\right)^{2} \leq \pi^{2}\left\{\left(\int_{\alpha}^{\infty}\left(e^{-(x-\alpha)} A^{*}(x-\alpha)\right)^{2} d x\right)^{2}-\left(\int_{\alpha}^{\infty} \tilde{\omega}(x)\left(e^{-(x-\alpha)} A^{*}(x-\alpha)\right)^{2} d x\right)^{2}\right\}
$$

where $\tilde{\omega}$ is defined by (2.11).

Proof. First, we have the following relation:

$$
\int_{0}^{\infty} e^{-t} A^{*}(t x) d t=\int_{0}^{\infty} e^{-t} \sum_{n=0}^{\infty} \frac{a_{n}(x t)^{n}}{n !} d t=\sum_{n=0}^{\infty} \frac{a_{n} x^{n}}{n !} \int_{0}^{\infty} t^{n} e^{-t} d t=\sum_{n=0}^{\infty} a_{n} x^{n}=A(x) .
$$

Let $t x=s-\alpha$. Then we have

$$
\begin{aligned}
\int_{0}^{1} A^{2}(x) d x & =\int_{0}^{1}\left\{\int_{0}^{\infty} e^{-t} A^{*}(t x) d t\right\}^{2} d x=\int_{0}^{1}\left(\int_{\alpha}^{\infty} e^{-(s-\alpha) / x} A^{*}(s-\alpha) d s\right)^{2} \frac{1}{x^{2}} d x \\
& =\int_{1}^{\infty}\left(\int_{\alpha}^{\infty} e^{-(s-\alpha) y} A^{*}(s-\alpha) d s\right)^{2} d y=\int_{0}^{\infty}\left(\int_{\alpha}^{\infty} e^{-(s-\alpha) u-(s-\alpha)} A^{*}(s-\alpha) d s\right)^{2} d u \\
& =\int_{0}^{\infty}\left(\int_{\alpha}^{\infty} e^{-(s-\alpha) u} f(s) d s\right)^{2} d u=\iint_{\alpha}^{\infty} \frac{f(s) f(t)}{s+t-2 \alpha} d s d t
\end{aligned}
$$

where $f(x)=e^{-(x-\alpha)} A^{*}(x-\alpha)$. By using (2.19), the inequality (3.6) follows from (3.8) at once. 


\section{Acknowledgment}

This work is a project supported by Scientific Research Fund of Hunan Provincial Education Department (07C520 and 06C657).

\section{References}

[1] G. H. Hardy, J. E. Littlewood, and G. Pólya, Inequalities, Cambridge University Press, Cambridge, UK, 1952.

[2] M. Gao and L. C. Hsu, "A survey of various refinements and generalizations of Hilbert's inequalities," Journal of Mathematical Research and Exposition, vol. 25, no. 2, pp. 227-243, 2005.

[3] M. Gao and B. Yang, "On the extended Hilbert's inequality," Proceedings of the American Mathematical Society, vol. 126, no. 3, pp. 751-759, 1998.

[4] B. Yang and L. Debnath, "On a new generalization of Hardy-Hilbert's inequality and its applications," Journal of Mathematical Analysis and Applications, vol. 233, no. 2, pp. 484-497, 1999.

[5] K. Jichang and L. Debnath, "On new generalizations of Hilbert's inequality and their applications," Journal of Mathematical Analysis and Applications, vol. 245, no. 1, pp. 248-265, 2000.

[6] L. He, M. Gao, and S. Wei, "A note on Hilbert's inequality," Mathematical Inequalities and Applications, vol. 6, no. 2, pp. 283-288, 2003.

[7] Y. Jin, Applied Integral Tables, Chinese Science and Technology University Press, Hefei, China, 2006. 